\title{
Acceleration of Images via Software and Hardware using Proprietary Tools \& Open Sources for Healthcare Industry
}

\author{
Garima Sharma \\ Department of Electronics and Communication Engineering, Bhagat Phool Singh Mahila Vishwavidyalaya, Khanpur \\ Kalan, Sonipat, Haryana, India \\ Email: firstgarima4@gmail.com \\ Krishan Kumar \\ Department of Electronics and Communication Engineering, Bhagat Phool Singh Mahila Vishwavidyalaya, Khanpur \\ Kalan, Sonipat, Haryana, India \\ Email: xs2krishan@yahoo.co.in
}

\begin{abstract}
Medical imaging appliances play a pivotal role in preventive medicine as the industry combat to low patient expense and acquire early disease estimation using nonintrusive methods. There are proprietary software packages which provide fast development for designing image processing algorithms. Another trend is to use open source softwares. With the advancement of VLSI (Very Large Scale Integration) technology, hardware implementation has also become an alternative. Proprietary hardwares provide flexibility, efficient power and timing constraints whereas open source hardwares provide optimum quality and cost constraints. The Present study is useful for image architects, researchers, biologists to learn various proprietary and open sources softwares as well as hardwares utilized for distinct applications of the healthcare industry.
\end{abstract}

Index Terms-BioimageXD, XSG (Xilinx system generator), Paraview, Raspberry pi, Panda boards, FPGA (Field-programmable gate array).

\section{INTRODUCTION}

Digital image processing is used in every sphere of life such as remote sensing, surveillance, agriculture, food industry and medical etc. Medical image processing is an emerging field in order to provide health monitoring. Image architects use proprietary tools which include Matlab/Simulink (The Mathswork Inc.) and LabVIEW (National Instruments Corporation).Matlab is used for algorithmic development and Simulink for system level design. Another graphical modeling tool is LabVIEW which provide high resolution with low latency. These proprietary tools are also utilized for hardware implementation of images. Biologist uses open source medical toolkits in order to detect artifacts. These toolkits are not developed for hardware implementation. Another option is to use general purpose open sources for image processing such as Scilab (The Scilab consortium) and OpenCV (Open Source Computer Vision) [6] which are compatible via software as well as hardware level implementation. Most of the researchers use FPGA boards [2] for hardware implementation of images due to its accuracy while rest uses open sources hardwares [20] such as Arduino, Beagle board [21], Panda board [45] and Raspberry Pi board [5] due to its cost effective constraints. The best option is to use a combination of open source software with open source hardware.

Medical images consist various modalities such as MRI (medical resonance imaging) which uses magnetic field with radio frequencies introduced into it, CT (computed tomography) and PET (positron emission tomography) used for cancer diagnosis. These modalities provide grayscale images [41]. A combination of computed tomography with PET (PET/CT) also plays an important role in medical imaging. Apart from these, there are other functional imaging modalities exist which are used for detection of diseases such as lung cancer. These include Diffusion-weighted magnetic resonance imaging (DW-MRI), dynamic enhanced CT (DCE-CT), and (FDG-PET/CT) integrated positron emission tomography and CT with F-FGD (fluorodeoxyglucose). DCE-CT is used for visualization and measurement of blood flow, volumes, tumors with high accuracy while DW-MRI shows water molecules diffusion within the tissues, FDG-PET/CT describes the metabolisms of tumors. Hence, these functional imaging modalities are very crucial in the field of disease detection [36]. This medical imaging is used to create images of a patient body which consist different modalities which are very unique. Image processing has most prominent applications in the field of medical [27]. It includes the application areas in which information is extracted from the medical images [44]. As medical images obtained from various cameras which consist various types of noises and complexity [46]. Hence medical image processing is used to achieve better results which involve various operations. This approach deals with medical images by applying different algorithms at different stages. It includes segmentation in order to extract stones, 
cancer cells and tumors and enhancement in order to improve the quality of images [37].

\section{Medical Image Processing Via Software}

There are various tools which can be utilized for medical image processing as depicted in figure 1. Proprietary tools provide fast development, accuracy, flexibility and speed constraints. Open source softwares are those whose source code can be modified or enhanced by anyone. These are free of cost while proprietary softwares are very costly and also it consists some restrictions regarding license availability.

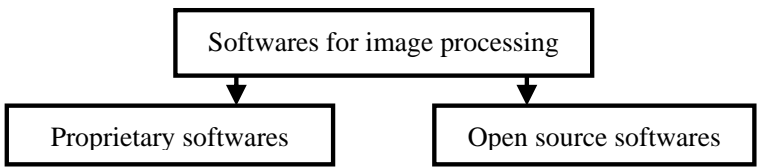

Fig.1. Classification of softwares used for image processing

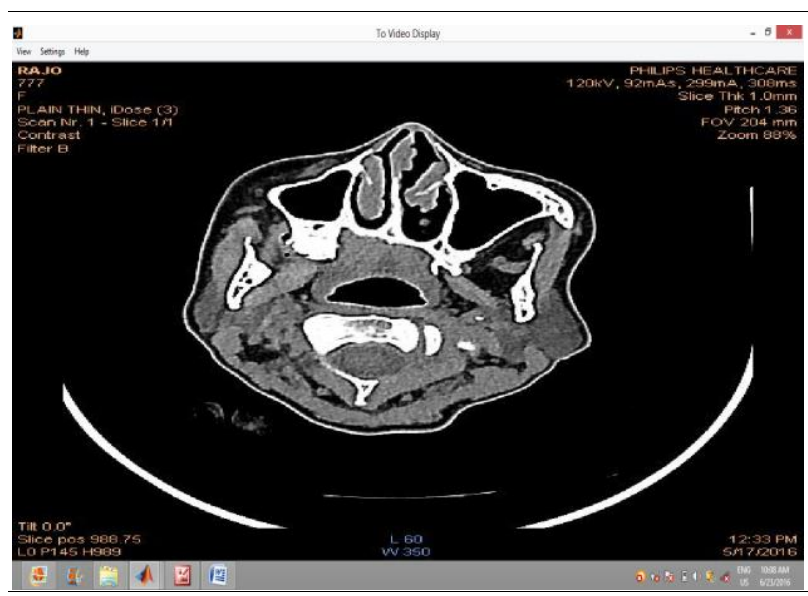

(a) Input image

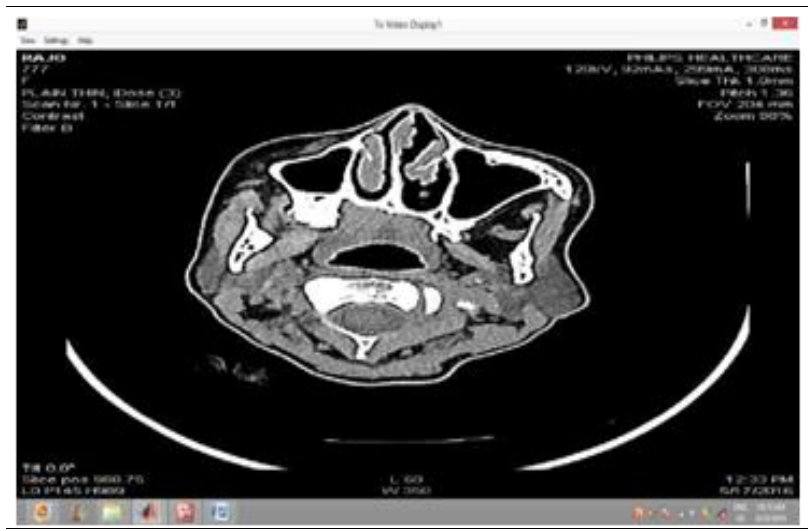

(c) Image enhancement

\section{A. Proprietary Softwares for Medical Image Processing}

Medical image processing uses proprietary tools to design various algorithms. These proprietary tools are Matlab/Simulink and LabVIEW. These tools are widely utilized in distinct applications of image processing.

Image processing using Matlab can be done in two ways either by using programming which includes script file or by using computer vision library of Simulink in which models are designed. Matlab is used by image architects in order to analyze images quantitatively and qualitatively [31]. It involves various operations like preprocessing of images in order to remove noise and artifacts from the images. Various algorithms of image processing such as image inversion, enhancement [57], segmentation [42] algorithms implemented using Matlab are depicted in figure 2 .

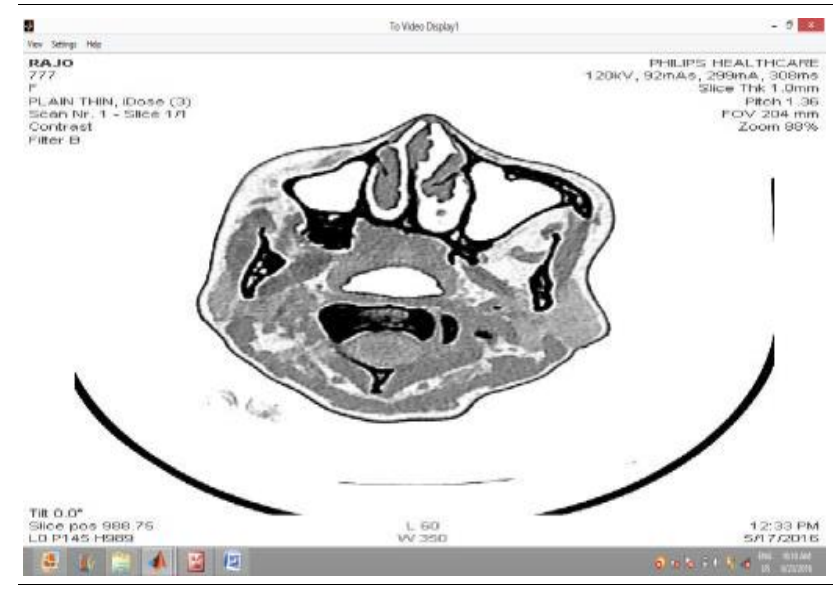

(b) Image inversion

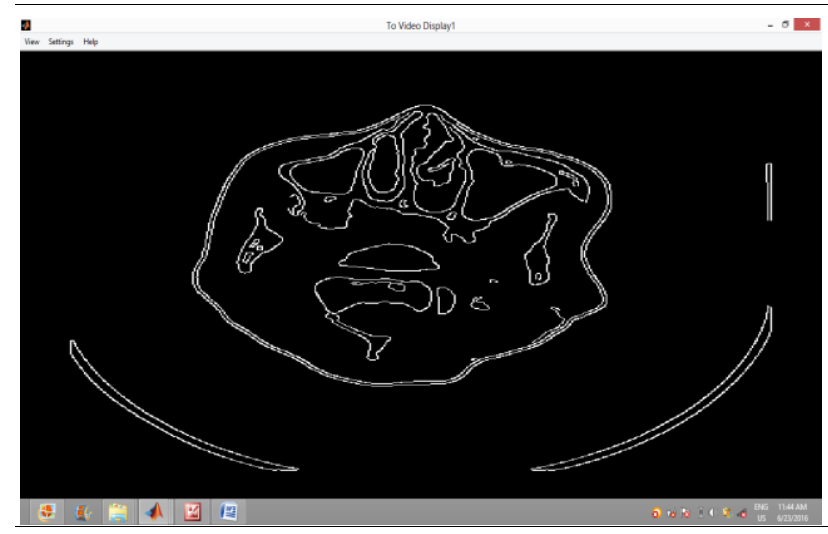

(d) Edge detection

Fig.2. (a-d): Image processing using Matlab

Another option is to use LabVIEW which consist some special tools for image processing [60]. IMAQ vision toolbox in LabVIEW which is a part of NI Vision contains a complete set of image acquisition and image processing functions which makes image processing more efficient as no programming is required. It includes various blocks like IMAQ read image and IMAQ create image block in order to design models for image processing. IMAQ toolbox is very efficient for applications of image processing. Vision assistant can 
also be used for developing image processing algorithms. In this performance can be analyzed by calculating statistical parameters and these parameters can also be
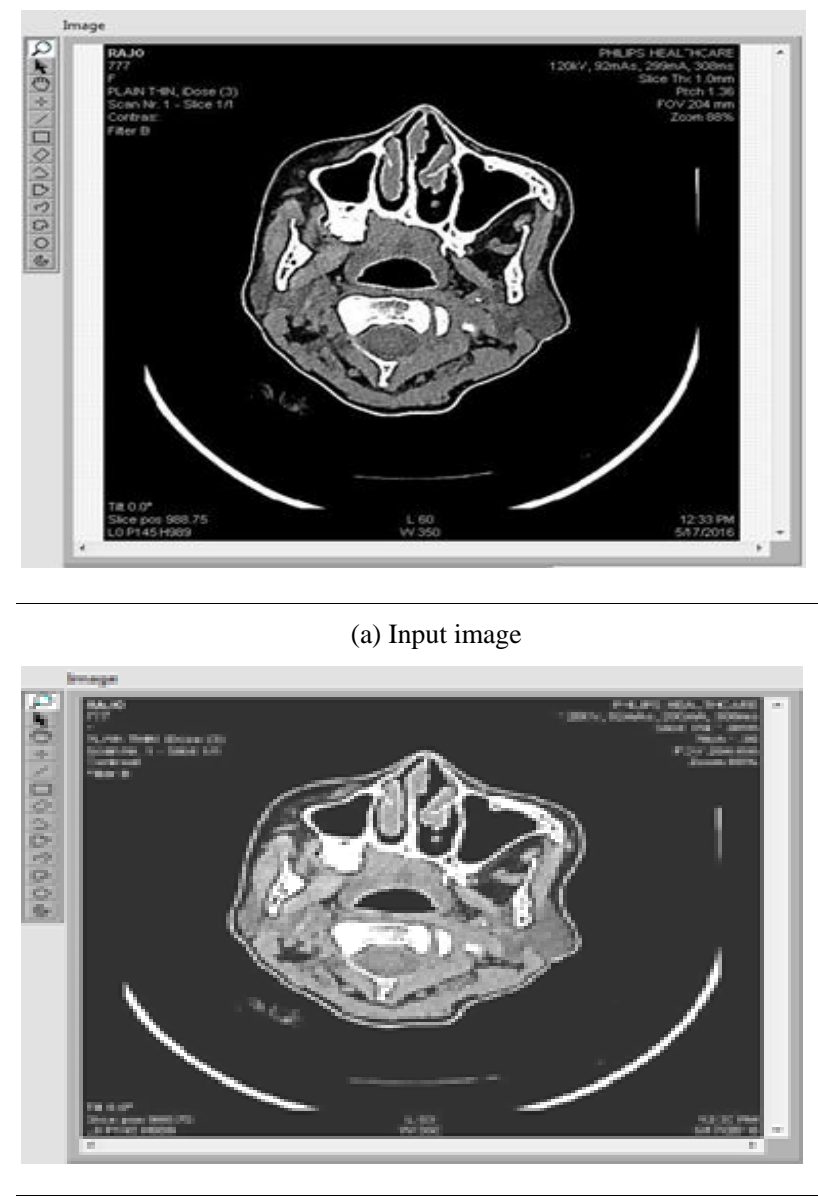

(c) Image enhancement analyzed from line profile in vision assistance. Statistical parameters can also be analyzed using IMAQ toolbox. Image processing using LabVIEW is depicted in figure 3.

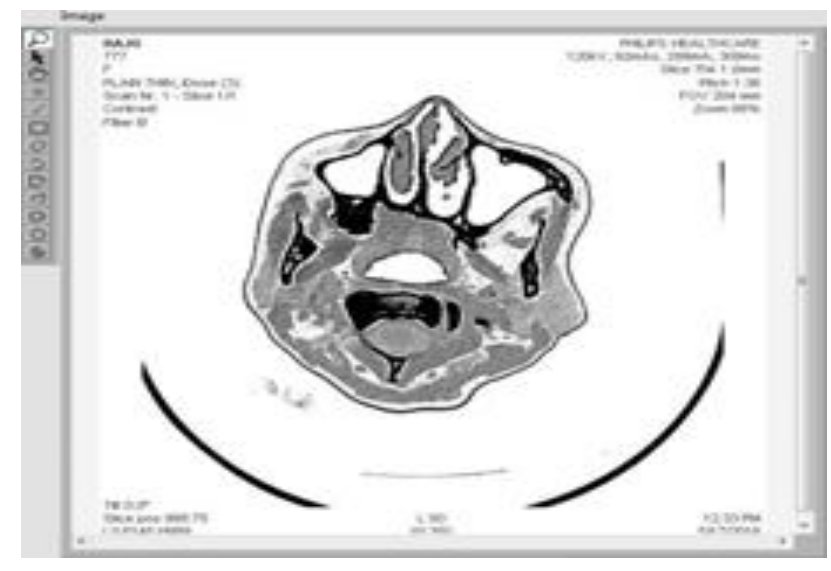

(b) Image inversion

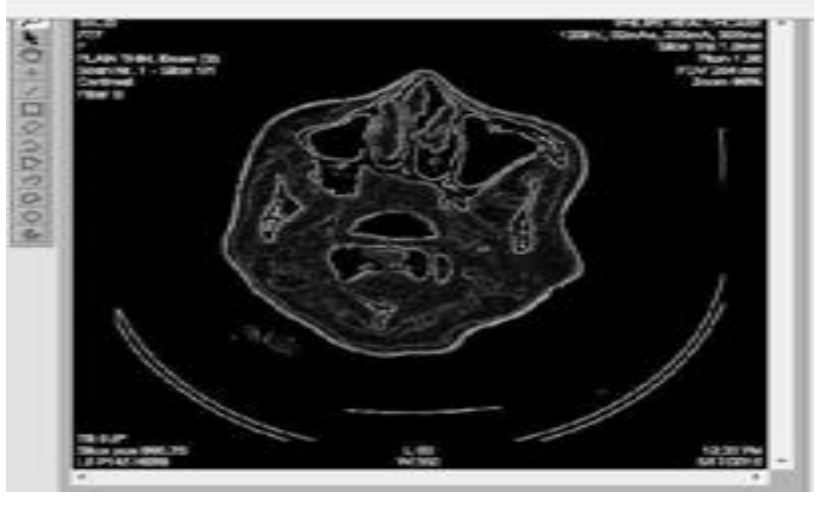

(d) Edge detection

Fig.3. (a-d): Image processing using LabVIEW

Proprietary tools such as Matlab [16] and LabVIEW are very efficient for designing image processing algorithms. Image architects utilized these tools for distinct applications of image processing. These tools can interact with some open source tool. These tools are more popular because of their accuracy, flexibility and timing constraints. These tools are also utilized for hardware implementation of images.

\section{B. Open Source Toolkits for Medical Image Processing}

Open source softwares provide accurately and cost effective access to the scientist in order to provide the wealth of information. The source code of these tools can be enhanced or modified by anyone. Open source toolkits are used to meet constraints such as faster feature implementation, free of cost, fast fixes for security, multiple options for a given task and fast upgrades to new releases. Open source tools for image processing are capable of performing basic operations on images such as image inversion, enhancement, compression, fusion, segmentation etc along with some additional features. These tools are divided into two categories as depicted in figure 4. General purpose open source tools are applicable for distinct applications whereas application specific open source tools are applicable for a specific application.

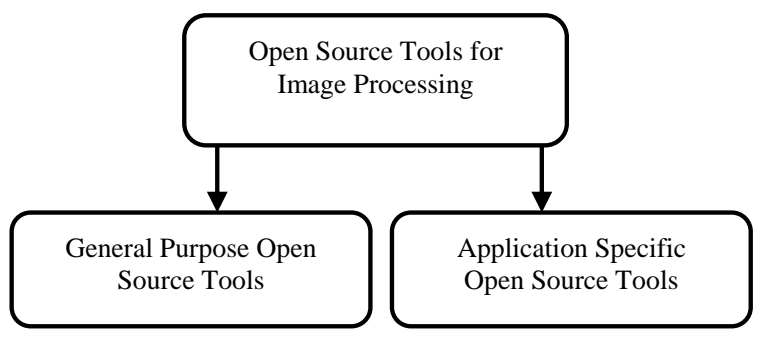

Fig.4. Classification of open source tools for image processing

General purpose open source tools: General purpose open source tools are those which are generally used for various applications. For image processing, open source tools such as Scilab and OpenCV [28] are general purpose open sources which are used to design algorithms for segmentation, enhancement etc for 
different applications. These softwares are applicable in various fields such as food industry, medical, agriculture, remote sensing etc. Scilab is open source software which can be utilized for image processing at the software level.

There are three open source toolkits used in Scilab for designing image processing algorithms which are SIP

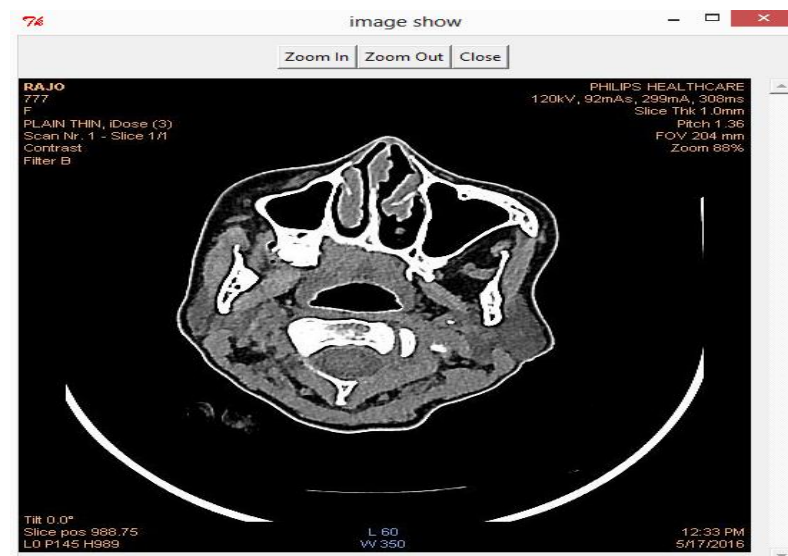

(a) Input image

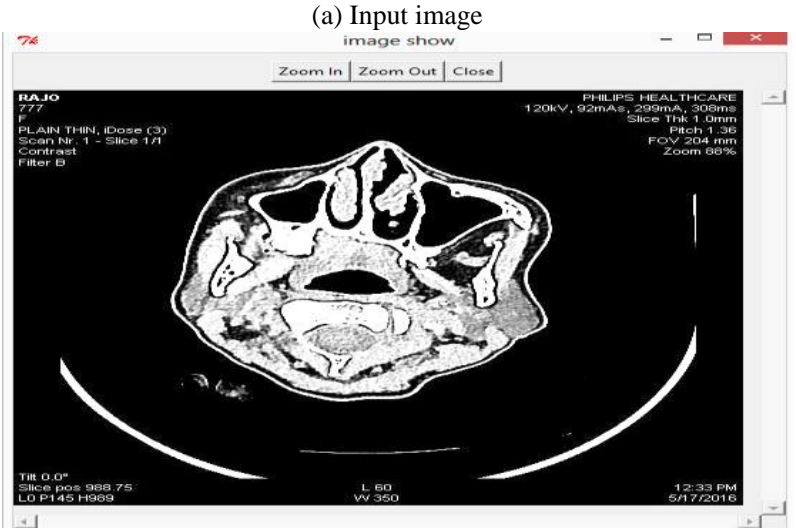

(c) Image enhancement
(Scilab Image Processing), IPD (Image processing design) toolbox and SIVP (Scilab Image and Video Processing). SIVP is based on OpenCV [15]. Image processing using SIVP is as depicted in figure 5.

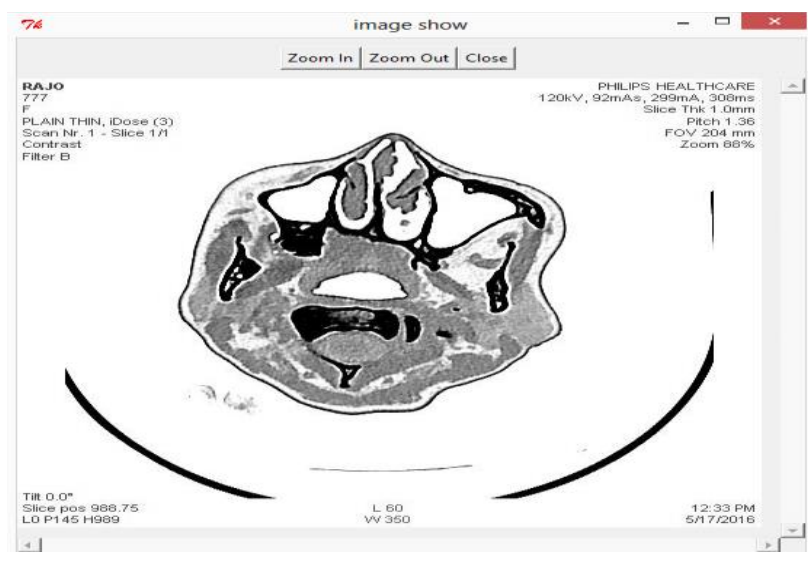

(b) Image inversion

image show

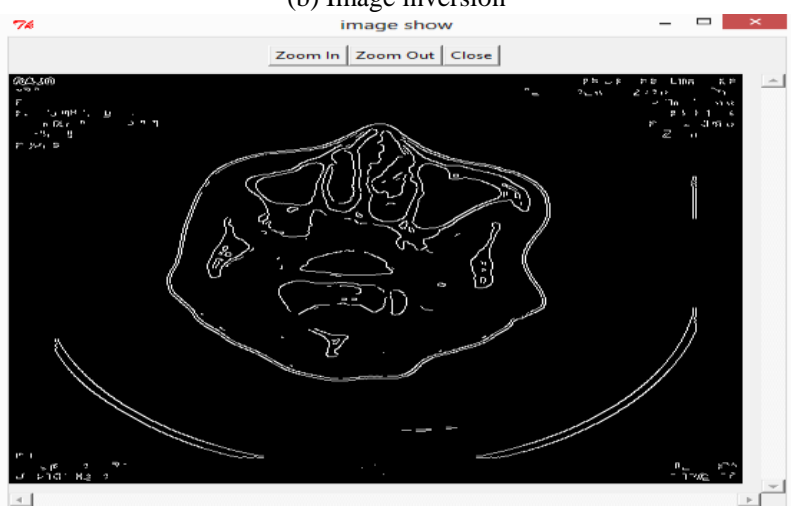

(d) Edge detection

Fig.5. (a-d): Image processing using SIVP (Scilab Image and Video Processing) toolkit

Table1. Open source medical image analysis softwares

\begin{tabular}{|c|c|c|c|}
\hline Sr. no. & Software & Applications & Reference \\
\hline 1 & VTK & Commercial applications of medical and research and development & www.vtk.org \\
\hline 2 & ITK & Visualization, analysis, image-guided surgery applications & Www.itk.org \\
\hline 3 & FSL & Analysis of brain imaging data & fsl.fmrib.ox.ac.uk/fsl/fslwiki/FSL \\
\hline 4. & SPM & Analyze data of brain imaging & www.fil.ion.ucl.ac.uk/spm/ \\
\hline 5 & GIMIAS & Filtering, segmentation and extraction of region of interest & www.gimias.org \\
\hline 6 & 3D Slicer & $\begin{array}{l}\text { Image registration and builds surface models from image labels, automatic } \\
\text { segmentation, 3D visualization }\end{array}$ & www.slicer.org \\
\hline 7 & MIA & Performs various tasks on 2D, 3D images & http://mia.sourceforge.net \\
\hline 8 & Camino & Brain imaging in order to detect brain disorders & http://cmic.cs.ucl.ac.uk/camino \\
\hline 9 & ITK-Snap & Automatic segmentation in order to extract tumors in CT and MRI. & http://www.itksnap.org \\
\hline 10 & Paraview & $\begin{array}{l}\text { Multi-platform visualization, data visualization to analyze large data sets by using } \\
\text { distributed memory resources }\end{array}$ & www.paraview.org \\
\hline 11 & VV & $\begin{array}{l}\text { Visualization of 2D, 3D, 4D images, performs operations such as fusion and } \\
\text { placement of landmark }\end{array}$ & $\begin{array}{l}\text { http://www.creatis.insa- } \\
\text { lyon.fr/rio/vv }\end{array}$ \\
\hline 12 & Fiji & Segmentation, registration, visualization, some advanced level operations & Fiji.sc/Fiji \\
\hline 13 & BioimageXD & Segmentation, filtering, visualization, qualitative analysis of images & www.bioimagexd.net/ \\
\hline 14 & elastix & Registration of medical images & http://elastix.isi.uu.nl/ \\
\hline 15 & MITK-DI & Visualization, reconstruction, pre-processing of diffused images & Mitk.org/wiki/DiffusionImaging \\
\hline
\end{tabular}


Application specific open source tools: These tools are designed for some specific applications. For example, the specially designed toolkit for medical is not applicable in the field of agriculture and remote sensing etc. For medical image processing, there are some specially designed toolkits which performed operations on medical images. It also performs some additional operations on images which are beneficial for detecting diseases.

Application specific open source tools for medical image processing are of three types- programming based, Simulation-based and GUI based [61]. Some open

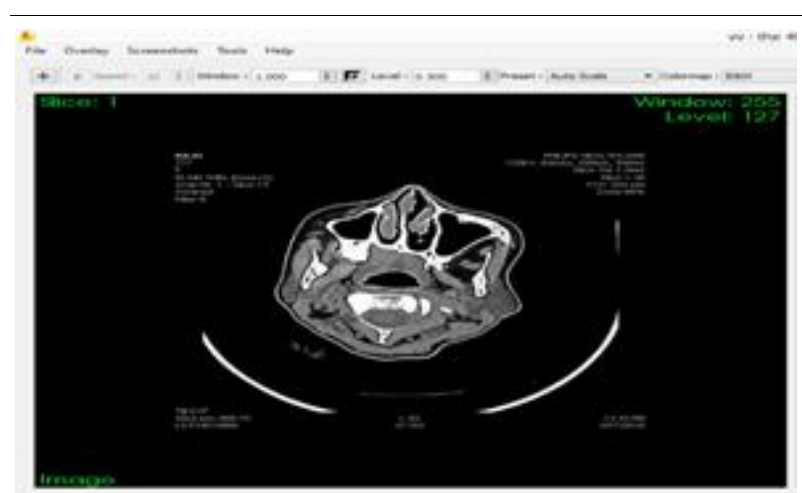

(a) Input image

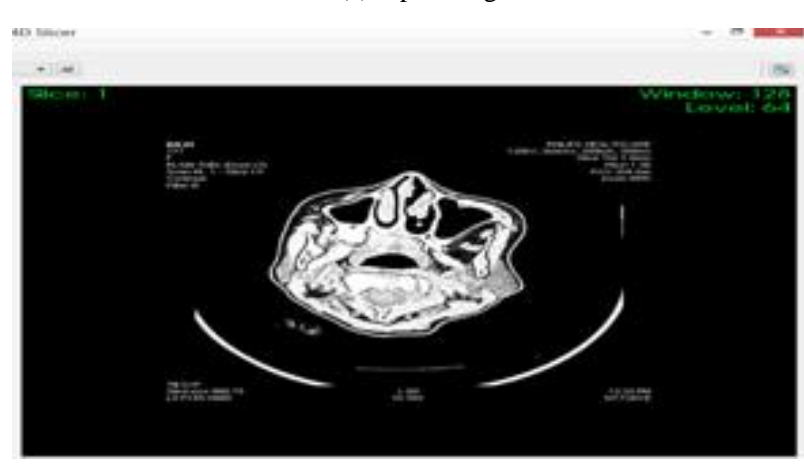

(c) Image enhancement sources are in the form of toolkits and these toolkits can be interfaced with MATLAB/Simulink. Some of the open sources toolkits used for medical image analysis are as depicted in table 1 .

These toolkits are used by biologists in order to detect diseases. These toolkits performed various image processing operations along with some additional operations which are required for distinct biomedical applications. Image processing using medical toolkit VV [62] which is 4D slider is as depicted in figure 6.

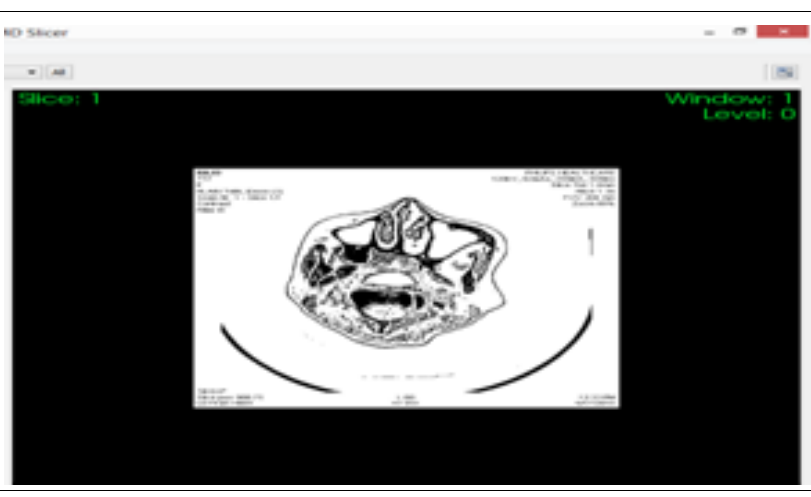

(b) Image inversion

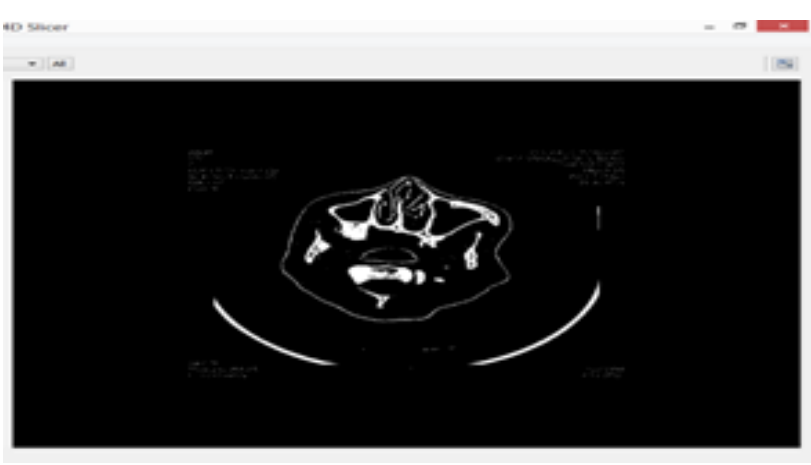

(d) Edge detection

Fig.6. (a-d): Image processing using VV (4D Slicer)

The open source softwares can also perform the same operations which can be performed using proprietary tools. Biologists directly utilize these open sources toolkits for medical applications in order to detect abnormalities and disease.

\section{Medical Image Processing Via Hardware}

Hardware implementation has become a very attractive alternative with the advancement in the VLSI (Very Large Scale Integration) technology [9]. Hardware implementations consist parallel and pipeline architecture which reduces the execution time [29].
Hardware implementation of images focuses on cost, time and power parameters [30]. There are various technologies available for implementing images. Hardware implementation is used in order to meet constraints such as high speed, feasibility, less time consumption, possibility of Simulation of logic input on hardware and co-simulation of already simulated logic [34]. It helps in the reformulation of the algorithm, minimizes time-to-market cost and provides debugging and prototyping [35]. Hardware used for image processing is of two types such as proprietary hardwares and open source hardwares [39]. Different types of hardwares utilized for implementation of image processing algorithm are depicted in figure 7. 


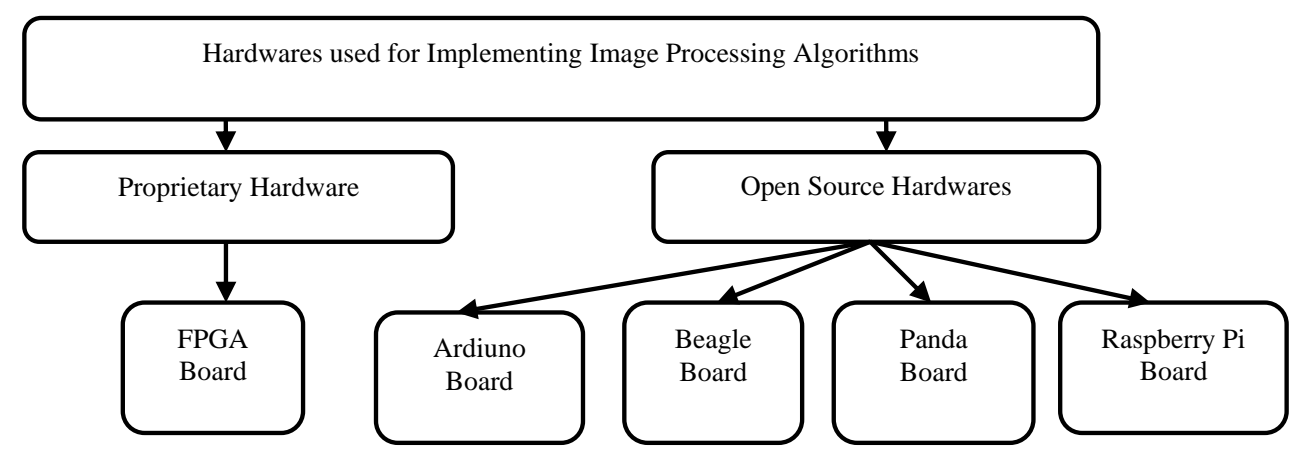

Fig.7. Types of hardwares used for implementing image processing algorithms

\section{A. Proprietary Hardware for Medical Image Processing}

FPGA [3] is a proprietary hardware which is highly preferable for implementation due to its small size, low power consumption. Image architects generally use proprietary hardware for image processing. Matlab and LabVIEW are utilized by image architects for hardware implementation of images.
Architects prefer Matlab for Hardware implementation using FPGAs [4]. FPGA implementation can be done either by using Xilinx System Generator (XSG) or FPGA in the loop (FIL) [10]. Xilinx creates a library in Simulink called Xilinx system generator which generates VHDL code [12]. Simulink model for image processing algorithms using blocks of XSG library [18] along with output image is depicted in table 2.

Table 2.FPGA implementation of image processing algorithms

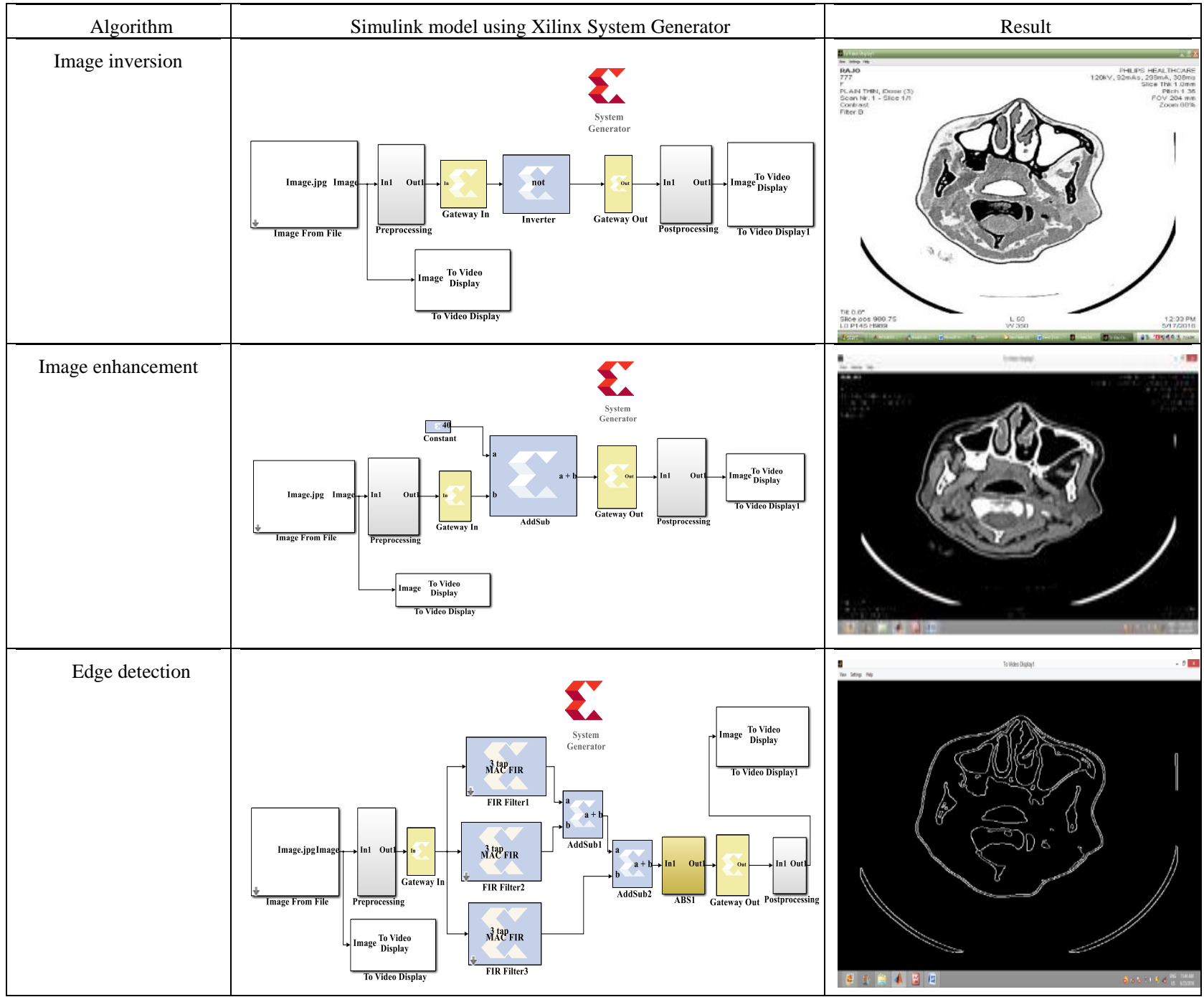


The merit of using Xilinx system generator is that the architects can estimate the utilized components of FPGA [14]. The device utilization summary provides the information regarding components used for designing algorithms of image processing [58]. Device utilization summary for edge detection algorithm is as shown in table 3.

Table 3. Device utilization summary of edged detection using Xilinx System Generator

\begin{tabular}{|c|c|c|c|c|c|c|}
\hline Sr. no. & Image processing algorithms & Slices & Flip Flops & LUTs & IOBs & GCLKs \\
\hline 1 & Image inversion & 1 & 1 & 16 & 33 & 1 \\
\hline 2 & Image enhancement & 1 & 1 & - & 41 & 1 \\
\hline 3 & Edge detection & 235 & 443 & 181 & 44 & 1 \\
\hline
\end{tabular}

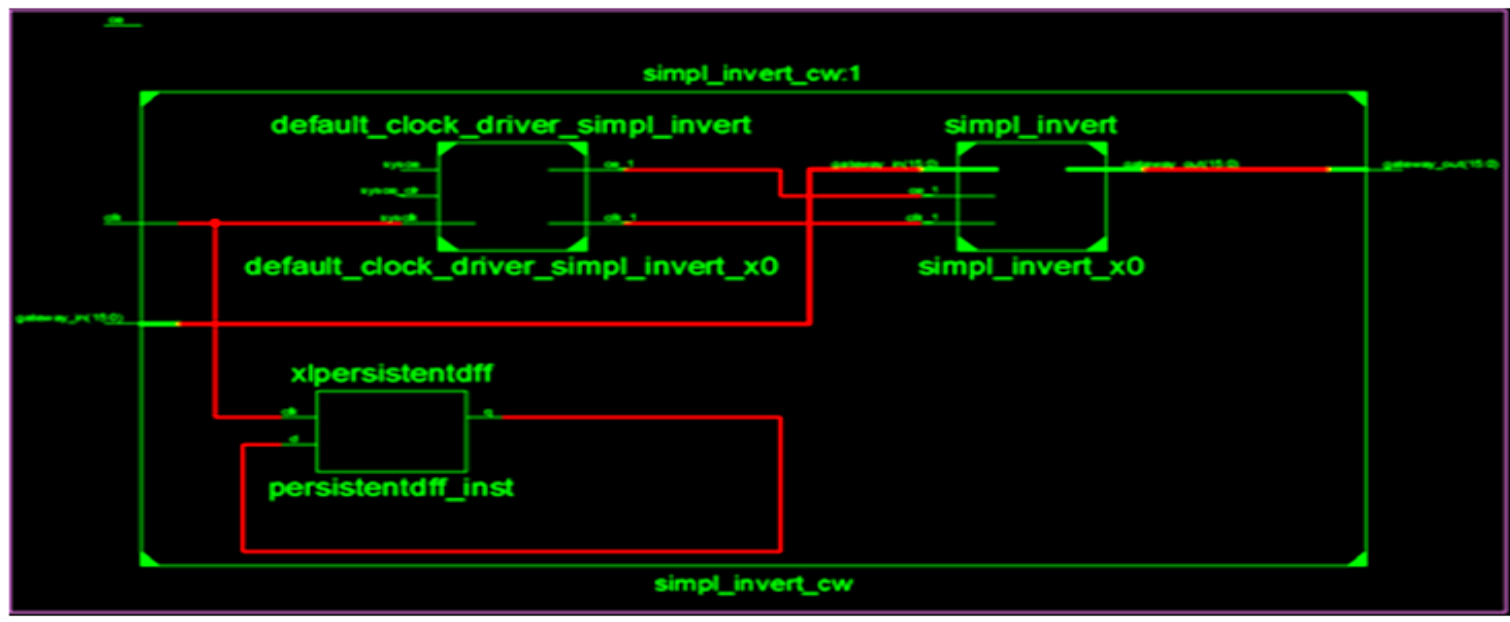

(a) RTL view of image inversion

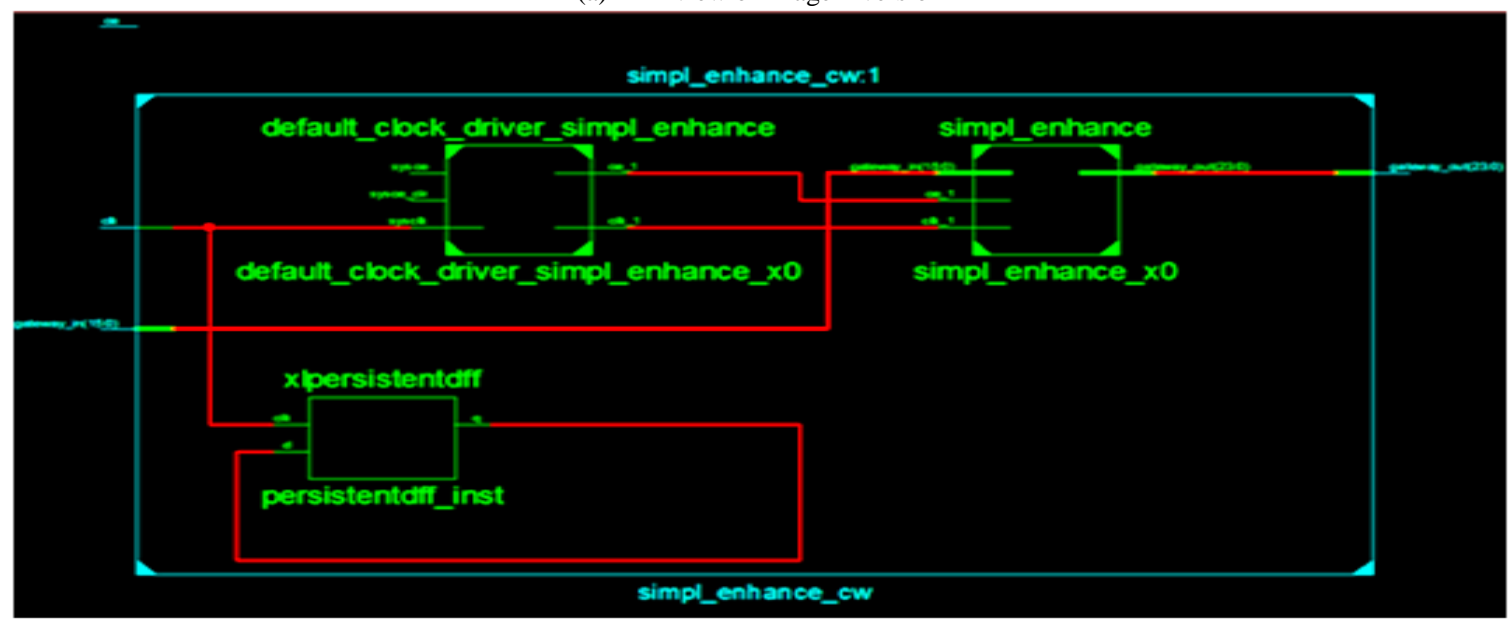

(b) RTL view of image enhancement

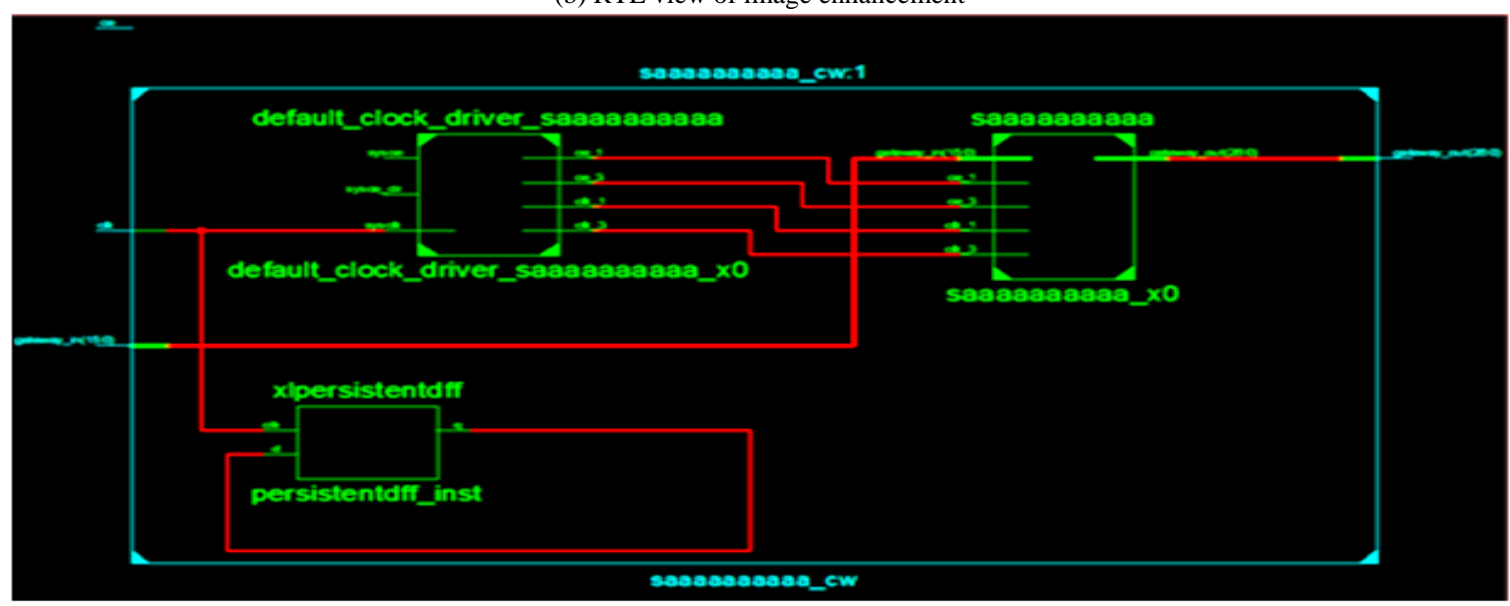

(c) RTL view of edge detection

Fig.8. (a-c): Register Transfer Logic (RTL) view of image processing algorithms 
Xilinx system generator is a mapping tool as it provides hardware software co-simulation [19]. RTL (Register Transfer Logic) view is a schematic representation in terms of gates, adders, multipliers etc [26]. RTL view of image processing algorithms is depicted in figure 8.

RTL view helps to discover design issues at the early stage [24]. Hardware implementation of medical image processing can also be done by using LabVIEW. In the case of LabVIEW, it is not possible to directly interact with FPGA. Hence image architects utilize Zed board and Zynq 7000, MyRIO [7] for hardware implementation of images It requires the installation of vision development module with NI LabVIEW FPGA module. Image processing algorithms which utilize a minimum number of resources, minimum time along with minimum power is very efficient for designing equipment for healthcare sector [22].

B. Open Source Hardware for Medical Image Processing

Hardware implementation using open source hardwares such as beagle board, panda board, and raspberry pi boards [25] can be done by using Simulink. Simulink provides built-in support for running and testing

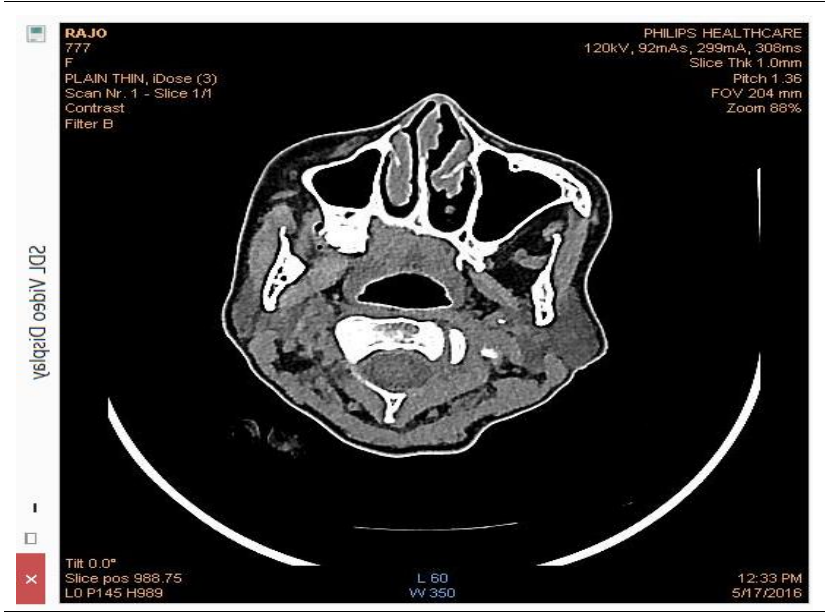

(a) Input image

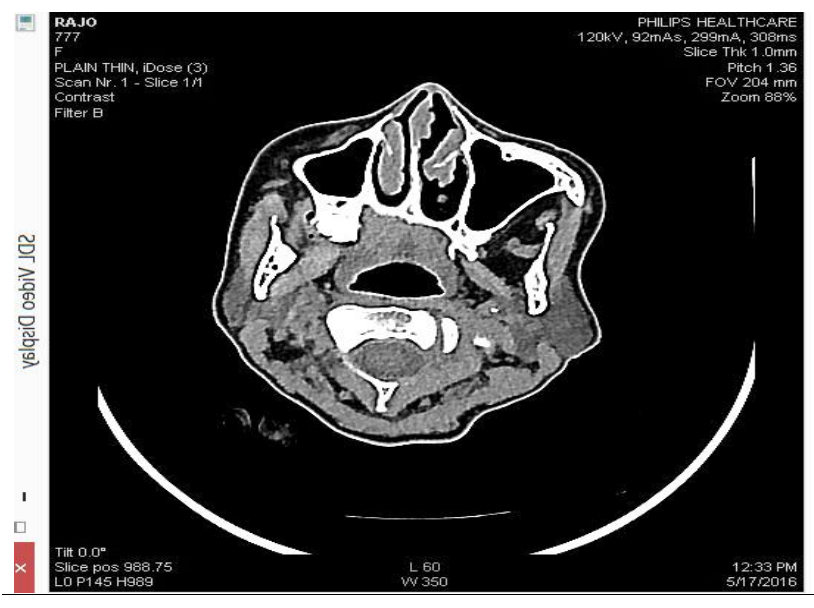

(c) Image enhancement image processing algorithms on these low-cost image processing hardwares. For hardware implementation using beagle board [40], architects use Matlab2012a or later versions. For this, an additional library needs to be installed. Simulink model for image processing algorithms using beagle board is depicted in figure 9 .

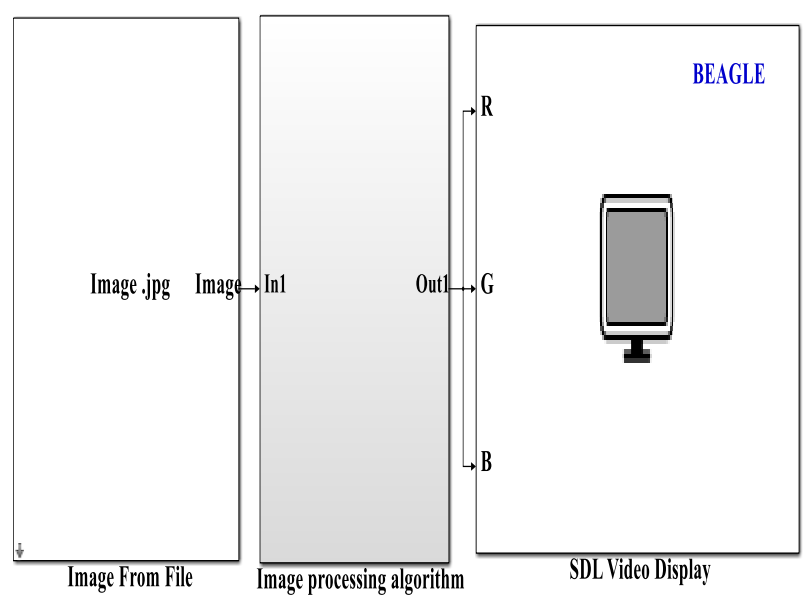

Fig.9. Simulink model of image processing algorithms using beagle board [32]

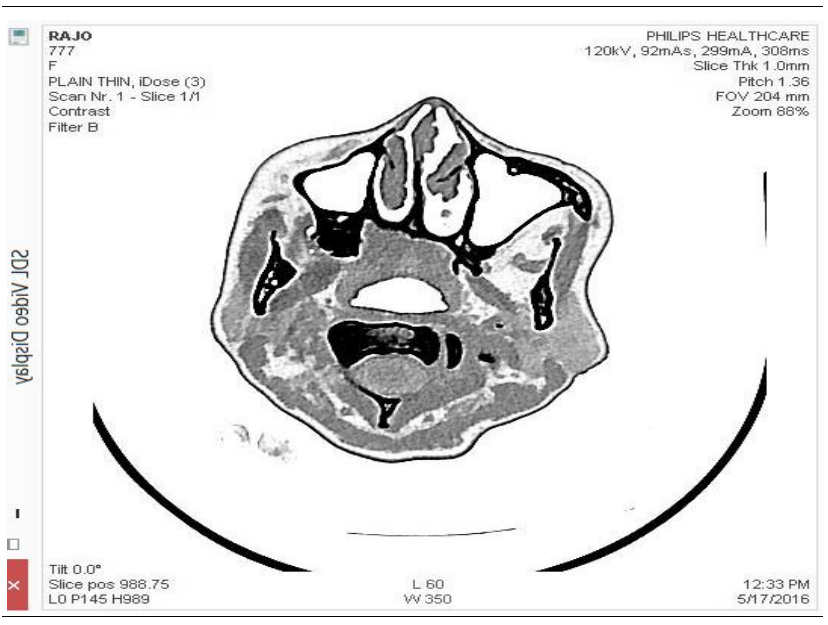

(b) Image inversion

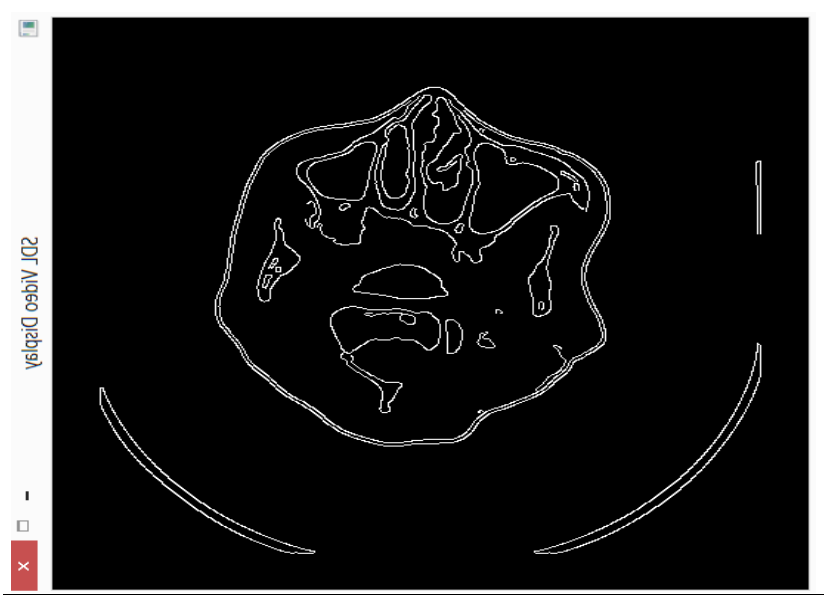

(d) Edge detection

Fig.10. (a-d): Image processing using beagle board [49] 
In order to analyze results using open source hardwares the output of SDL video display needs to be rotated and flipped. Image processing algorithms such as image inversion, image enhancement, edge detection using Simulink support package for beagle board [49] hardware library and computer vision library is depicted in figure 10.

Matlab2012b is utilized by architects for implementing image processing algorithms using panda board. Its implementation is similar to beagle board. For implementation using Arduino, architects need to generate $\mathrm{C}$ code using Simulink.

Raspberry Pi [8] is a credit card size small hardware used for image processing. It is used in various applications of image processing. For implementing image processing algorithms on raspberry pi, MATLAB 2013a or above version is required. Simulink has inbuilt support for raspberry pi hardware [11]. For this Simulink support package for raspberry pi hardware, a library needs to be installed. Simulink model for implementation of image processing algorithm consist of implementing

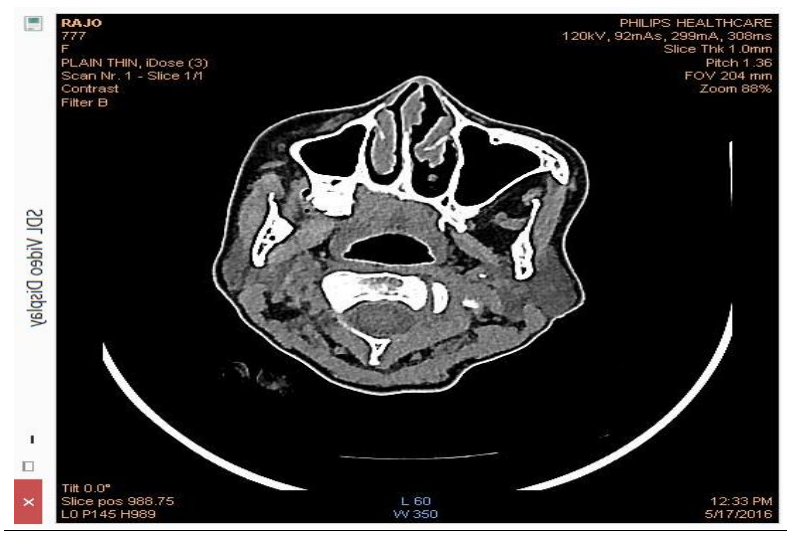

(a) Input image

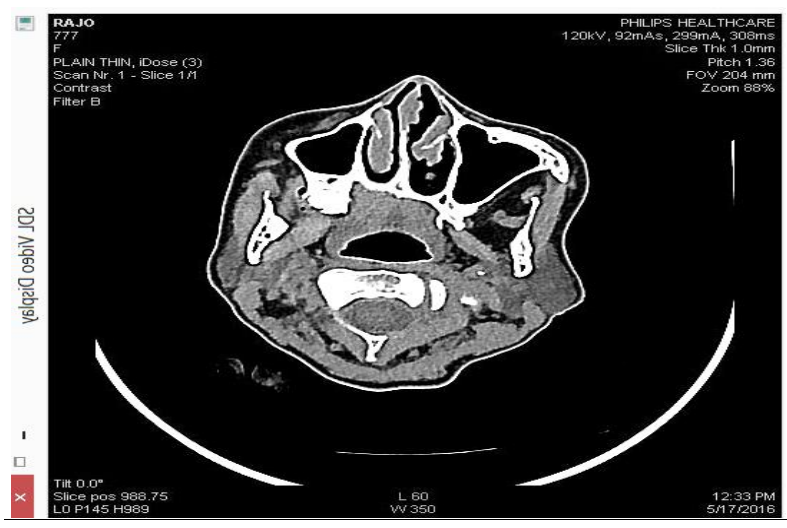

(c) Image enhancement image processing algorithms using raspberry pi board [13] is depicted in figure 11 .

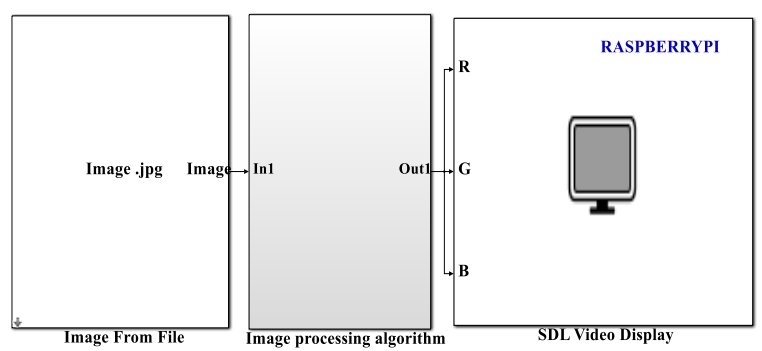

Fig.11. Simulink model of image processing algorithms using beagle board [33]

Image processing algorithms using Simulink support package for raspberry pi hardware library and computer vision library is as depicted in figure 12 .

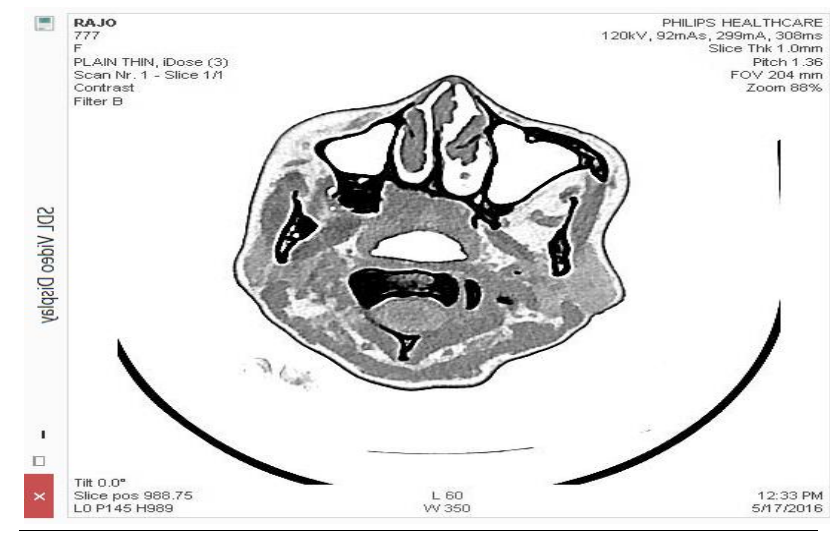

(b) Image inversion

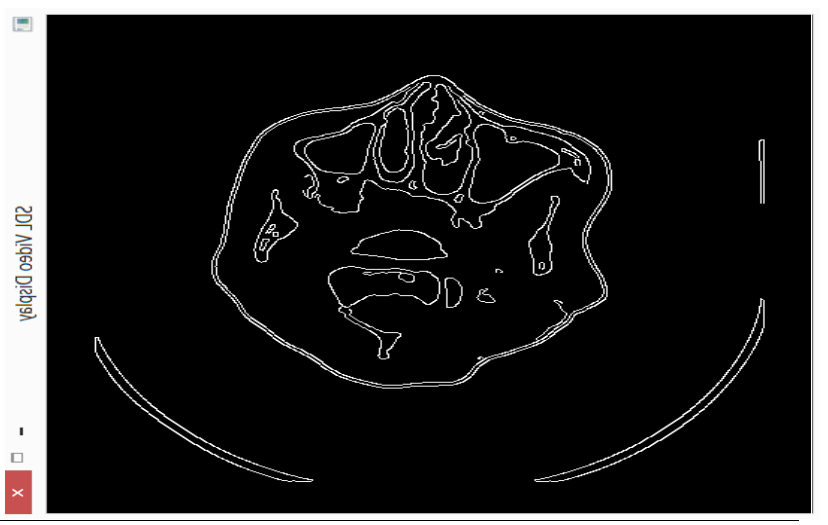

(d) Edge detection

Fig.12. (a-d): Image processing using raspberry pi [43] 
Open source hardwares such as beagle board [1] and raspberry pi board provides cost effective framework along with optimum quality.

\section{CONCLUSION}

Healthcare industry design CT scan, MRI and Ultrasound machines which are used by radiologists in order to detect artifacts and abnormalities in the human body. These equipment consist image processing algorithms. Image architects use proprietary tools such as Matlab and LabVIEW for implementing image processing algorithms. In this study, we have used computer vision library of Simulink in order to design image processing algorithms. The IMAQ vision toolbox is utilized for performing image processing algorithms using LabVIEW. LabVIEW provides a very fast implementation. Hence it is very efficient for real-time implementation. Matlab/Simulink has built-in support for hardware implementation. It is very efficient for hardware implementation of images. In the case of LabVIEW, it is not possible to directly interact with FPGA. Hence image architects utilize Zed board and Zynq 7000 for hardware implementation of images. So we have concluded that proprietary tools are very efficient for implementing image processing algorithms. The cost of these proprietary tools is very high and also sometimes compatibility difficulties arise due to technical changes in the new release. Due to the high cost of these softwares, healthcare industry does not provide the cost effective solution to the rural and economically backward people. Hence in order to achieve cost-effective constraints along with optimum quality open source tools can be utilized. In this we have used Scilab image and video processing (SIVP) toolkit which is a general purpose tool for designing image processing algorithms. We have concluded that SIVP is very efficient for implementation of images via software. But still, it is not developed for hardware implementation. OpenCV is very efficient software for implementation of images via software and hardware. So these tools are can be utilized by image architects for developing image processing algorithms. The proprietary tools such as Matlab, LabVIEW, general purposes open sources such as Scilab and OpenCV are used only by image architects but not by biologists. Hence there are some special toolkits which are designed for biologists in order to detect abnormalities and diseases. These toolkits perform various additional operations which are required for the clinical purpose.

Medical equipment such as CT scan, MRI, Ultrasound machines consists FPGA and other DSP kits. FPGAs are used in medical equipment in order to meet constraints such as power efficiency, high speed etc. Image processing algorithms for FPGA using Xilinx System Generator (XSG) provides flexibility, efficiency, speed constraints. It utilizes a limited number of resources at maximum frequency and minimum power which is very efficient for designing equipment for the healthcare industry. In order to meet cost-effective constraints along with optimum quality, open source hardwares are also a good option. There are various open sources DSP kits such as Arduino, beagle board, panda board and raspberry pi board. Simulink has built-in support for open source hardwares such as beagle board and raspberry pi board. Open source hardwares for image processing provide a cost effective framework along with optimum quality for the healthcare industry in order to provide betterment in health monitoring of rural and economically backward people.

\section{ACKNOWLEDGMENT}

Authors would like to thank BPS Government Medical College for Women, Khanpur Kalan, Sonipat for providing data and timely help in research. The work we present in this paper is completely supported by BPSMV, Khanpur Kalan, Sonipat, Haryana, India.

\section{REFERENCES}

[1] Garima Sharma, Krishan Kumar, "Prototyping of image enhancement algorithms using beagle board for rural health monitoring", International Journal of Advance Research in Science and Engineering (IJARSE), Volume 5, PP. 632-644, August 2016.

[2] Arun Mahajan, Paramveer Gill,"2D Convolution Operation with Partial Buffering Implementation on FPGA", International Journal of Image, Graphics and Signal Processing (IJIGSP), Volume 8, PP. 55-61, 2016.

[3] Siddarth Sharma, k. Pritamdas, "FPGA based efficient median filter implementation using Xilinx System Generator, International Journal of Innovative Research in Science, Engineering and Technology, Volume 5, PP. 8946-8952, May 2016.

[4] Durga Joshi, Richa Tiwari, "A study of VHDL implementation of fast sobel edge detection algorithm", International Journal of Innovative Research in Computer and Communication Engineering, Volume 4, PP. 97759777, May 2016.

[5] Swami Gururaj M., Naveen J., "Identification of counterfeit currency and denomination using Raspberry pi”, International Journal of Innovative Research in Electrical, Electronics, Instrumentation and Control Engineering, Volume 4, PP. 142-146, April 2016.

[6] Prof. Mangesh. T. Nikam, Mr. Jitendra. A. Patil, Mr. Vishal S. Patil, "Pedestrian Counting System Using Raspberry-Pi and OpenCV", International Journal of Innovative Research in Electrical, Electronics, Instrumentation and Control Engineering (IJIREEICE), Volume 4, PP. 94-96, March 2016.

[7] Smruti Ranjan Nayak, Shanaz Aman, Harish Chandra Mohanta, Dr Satyasish Miishra, "Design and implementation of real time video image edge detection system using MyRIO", International Journal of Advanced Research in Electrical, Electronics and Instrumentation Engineering, Volume 5, PP. 1793-1800, March 2016.

[8] Jyoti Yadav, Mohammed Firash Khanna, Muhammed Shahan K. K, Sreedevi p, Sumayya K, Manju V. M, "Raspberry pi based gesture photography", International Journal of Advanced Research in Computer and Communication Engineering,Volume 5, PP. 412-416, March 2016.

[9] Y. Premson, R. Sakthivel, "VLSI Architecture for Image Contrast Enhancement using Modified Adaptive Gamma Correction with Weighting Distribution”, Indian Journal 
of Science and Technology, Volume 9, PP. 1-7, February 2016.

[10] Mohammed Alareqi, Rachid Elgouri, Khalid Mateur, "Real time hardware co-simulation for image processing algorithms using Xilinx System Generator”, International Journal on Electrical Engineering and Informatics, Volume 7, PP. 711-723, December 2015.

[11] K. Horak, L. Zalud, "Image Processing on Raspberry Pi in Matlab", Advances in intelligent systems and computing, 4 November 2015.

[12] Ankita gupta, Himanshu Vaishnav, Himanshu Garg," Image Processing using Xilinx System Generator (XSG) in FPGA", International Journal of Research and Scientific Innovation (IJRSI), Volume 2, PP. 119-125, September 2015.

[13] Nirmal T. M., K. R. Joy, Rajeev K., "Implementation and optimization of connected component labeling in Raspberry Pi", ARPN Journal of Engineering and Applied Sciences, Volume 10, PP. 7670-7677, September 2015.

[14] S. R. Dixit, Dr. A. Y. Deshmukh, "Realization of canny edge detection algorithm using FPGA", International Journal of Engineering Sciences \& Research Technology (IJESRT), Volume 4, PP. 608-618, September 2015.

[15] Shubhashree Savant, "SIVP: SCILAB image and Video processing toolbox", CSI communication, Volume 39, PP. 37-38, September 2015.

[16] Shikha Maheshwari, Krishan Kumar, "Comparative analysis of lung cancer using different medical modality ", International Journal of Advanced Research in Computer and Communication Engineering, Volume 4, PP. 332-336, September 2015.

[17] Samar D. Gajbhiye, Pooja P. Gundewar," Object Tracking Using Embedded Platform for Video Surveillance”, International Journal of Innovative Research in Computer and Communication Engineering, Volume 3, PP. 67916798, July 2015.

[18] Kaji Ahmed Asif Fuad, Shahriyar Masud Rizvi, "Hardware software co-simulation of Canny edge detection algorithm", International Journal of Computer Applications, Volume 122, PP. 7-12, July 2015.

[19] K. Dinesh Kumar, A. V. karthik, K. Shravan Kumar, M. Koteswara Rao, V. Nancharaiah, "Implementation of sobel and prewitt edge detectors using Xilinx System Generator, International Journal of Advanced Engineering and Global Technology, Volume 3, PP. 901-905, July 2015.

[20] J. Manasa, J.T. Pramod, Dr. S. A .K. Jilani, Mr. S. Javeed Hussain, "Real Time Object Counting using Raspberry pi", International Journal of Advanced Research in Computer and Communication Engineering (IJARCCE), Volume 4, PP. 540-544, July 2015.

[21] V. Kamatchi Sundari, M. Manikandan, "Implementation of Multi Target Tracking Algorithm on Beagle BoardXm", Paripex-Indian Journal of Research, Volume 4, PP. 31-33, July 2015.

[22] Swati R. Dixit, Dr. A. Y. Deshmukh,“ Sobel Edge Detection Implementation using Spartan 3 FPGA and Xilinx System Generator", International Journal of Engineering Research and General Science, Volume 3, PP. 221-227, July-August 2015.

[23] Dushyant Mankar, Prof. S. S. Mungona, "Models for MATLAB and XSG Simulink Based Edge Detection Operations", International Research Journal of Engineering and Technology (IRJET), Volume 2, PP. 1254-1257, June 2015.

[24] Yogesh V. Raut, Charudatta V. Kulkarni, "FPGA Based Face Detection System using Xilinx System Generator",
International Journal of Advanced Research in Computer and Communication Engineering, Volume 4, PP. 423-426, June 2015.

[25] K. S. Shilpashree, Lokesha H, Hdimani Shivkumar, "Implementation of Image Processing on Raspberry Pi", International Journal of Advanced Research in Computer and Communication Engineering (IJARCCE), Volume 4, PP. 199-202, May 2015.

[26] Harshada Shimpi, Nisha Gaikwad, Meghana Dhage, Prof. A. S. Pawar," Implementation of Edge Detection Algorithm Using FPGA", International Journal of Advanced Research in Electrical, Electronics and Instrumentation Engineering, Volume 4, PP. 2193-2197, April 2015.

[27] Kalyani A. Dakre, Prof. P. N. Pusdekar , "Image Enhancement using Hardware co-simulation for Biomedical Applications", International Journal on Recent and Innovation Trends in Computing and Communication (IJRITCC) Volume 3, PP. 869-877, February 2015.

[28] Sharmila B, Karalan N and Nedumaran D, "Image Processing on DSP Environment Using OpenCV", International Journal of Advanced Research in Computer Science and Software Engineering (IJARCSSE), Volume 5, PP. 489-493, February 2015.

[29] Ms. P.H. Pawar, Prof. R. P. Patil, "FPGA Implementation of Canny Edge Detection Algorithm", International Journal of Engineering And Computer Science (IJECS), Volume 3, PP. 8704-8709, October 2014.

[30] K. Anil Kumar, M. Vijay Kumar, "Implementation of Image Processing Lab Using Xilinx System Generator ", Advances in Image and Video Processing, Volume 2, PP. 27-35, October 2014.

[31] K. Naresh, M. Mahender, "Implementation of image edge detection using canny edge detection algorithm", International Journal of Scientific Engineering and Technology Research, Volume 3, PP. 5840-5844, October 2014.

[32] Dr. S.A.K. Jilani, P. Mohammad Gouse, "Motion Detection Alert System using Beagle Bone Black", International Journal of Engineering Research \& Technology (IJERT), Volume 3, PP. 1249-1252, September-2014.

[33] Dr. S. A. K. Jilani, G.S.A. Manasa, "Raspberry Pi Based Color Speaker", SSRG International Journal of Electronics and Communication Engineering (SSRGIJECE), Volume 1, PP. 28-32, September 2014.

[34] Alareqi Mohammed, Elgouri Rachid, Hlou Laamari, "High Level FPGA Modeling for Image Processing Algorithms Using Xilinx System Generator”, International Journal of Computer Science and Telecommunications, ISSN 2047-3338, Volume 5, PP. 1-8, June 2014.

[35] Amiket A. Ingle, Vrushali G. Raut, "Hardware software co-simulation of edge detection for image processing system using delay block in XSG ", International Journal of Research in Engineering and Technology (IJRET), Volume 3, PP. 549-553, May 2014.

[36] Mohammed Yousuf Khan, Masarath Nayeem Tayyaba, M. A .Raheem, Ayesha Siddiqua and Syed Sameena, "Image Enhancement and Hardware Implementation of Edge Detected Vascular Images using Simulink Model", International Journal of Advanced Research in Computer and Communication Engineering, Volume 3, Issue 4, PP. 6385-6388, April 2014.

[37] Indrajeet Kumar, Jyoti Rawat and Dr. H.S. Bhadauria, “A Conventional Study of Edge Detection Technique in Digital Image Processing", International Journal of Computer Science and Mobile Computing, Volume 3, PP. 
328-334, April 2014

[38] Syed Sameer Rashid, Swati R. Dixit, A.Y. Deshmukh, "VHDL Based Canny Edge Detection Algorithm", International Journal of Computer Engineering and Technology, Volume 4, PP. 749-752, 1 April 2014.

[39] Pankaj Bhandari, Pankaj K Gupta, Karthik U. S Goutham Reddy, Jeeva. B, "Analysis of face recognition based on edge detection algorithm with hardware interfacing", International Journal of Advanced Research in Electrical, Electronics and Instrumentation Engineering, Volume 3, PP. 114-121, April 2014.

[40] Pradeep Kumar M, Lokesha H, "Real Time Implementation of Computer Vision Algorithms on Beagle board", IOSR Journal of VLSI and Signal Processing (IOSR-JVSP), Volume 4, PP. 1-6, MarchApril 2014.

[41] Hong Nguyen T. K., Cecile. Belleudy, Tuan. V. Pham, "Performance and Evaluation Sobel Edge Detection on Various Methodologies", International Journal of Electronics and Electrical Engineering, Volume 2, PP. 1520, March 2014.

[42] V. Kamatchi Sundari, M. Manikandan, P. Prakash, "FPGA Implementation of Sobel Edge Detector", International Journal of Advances in Science and Technology (IJAST), PP. 255-259, 15 February 2014.

[43] Md. Maminul Islam, Md. Sharif Uddin Azad, Md. Asfaqul Alam, Nazmul Hassan,"Raspberry $\mathrm{Pi}$ and image processing based Electronic Voting Machine (EVM)", International Journal of Scientific \& Engineering Research, Volume 5, PP. 1506-1510, January 2014.

[44] S W Harders, S. Balyasnikowa, B.M. Fischer, "Functional imaging in lung cancer", Clin Physiol Funct Imaging, Volume 34, pp. 340-355, 2014

[45] Parashar Dhakal, Nabin Sharma Rijal, Amar Mani Aryal, Ashutosh, Shrey Pratik, "Development of Real Time Area Based and Particle Filter Based Tracking on Panda Board", International Journal of Advanced Computer Engineering and Communication Technology (IJACECT), Volume 3, PP. 30-36, 2014.

[46] Prof. Kamal A. El Dahshan, Prof. Mohammed I. Youssef, Dr. Emad H. Masameer , Mohammed A. Mustafa, "Hardware Segmentation on Digital Microscope Images for Acute Lymphoblastic Leukemia Diagnosis Using Xilinx System Generator", International Journal of Advanced Computer Science and Applications(IJACSA), Volume 5, , PP. 33-37, 2014.

[47] M. Rathod, "Edge detection in VHDL", International Journal of Engineering Development and Research", Volume 2, PP. 1095-1099, 2014.

[48] Swapnil G. Kavitkar, Prashant L. Paikrao, "FPGA based image feature extraction using Xilinx System Generator", International Journal of Computer Science and Information Technologies, Volume 5, PP. 3743-3747, 2014.

[49] G. Johny and P. Vijay Daniel, "Real Time Embedded Implementation of Face Recognition Using Beagle Board", International Journal Of Engineering Sciences \& Research Technology (IJESRT), Volume 2, PP. 3452-3456, December 2013.

[50] Praveen vanaparthy, Sahitya. G, Krishna Sree, Dr. C. D. Naidu, "FPGA implementation of image enhancement algorithms for biomedical image processing", International Journal of Advanced Research in Electrical, Electronics and Instrumentation Engineering, Volume 2, PP. 5747-5753, November 2013.

[51] Ravi.s, Abdul Rahim. B, Fahimuddin shaik, "FPGA Based Design and Implementation of Image Edge Detection
Using Xilinx System Generator", International Journal of Engineering Trends and Technology (IJETT), Volume 4, PP. 4657-4660, Oct 2013.

[52] H.P. Narkhede, "Review of image segmentation technique", International Journal of Science and Modern Engineering (IJISME), Volume 1, PP. 370-372, July 2013.

[53] Ying shin, Weihua Zhu, "Medical Image Processing -A Machine Vision Based Approach", International Journal of Signal Processing, Image Processing and Pattern Recognition, Volume 6, PP. 139-146, June 2013.

[54] Neha. P. Raut, Prof. A. V. Gokhale, "FPGA Implementation for Image Processing Algorithms Using Xilinx System Generator", IOSR Journal of VLSI and Signal Processing (IOSR-JVSP), Volume 2, PP. 26-36, May - June 2013.

[55] Adhyana Gupta, "Hardware software co-simulation for traffic load computation using Matlab Simulink model blockset", International Journal of Computational Science and Information Technology (IJCSITY), Volume 1, PP. 112, May 2013.

[56] Shreedeep Gangopadhyay, Bhaskar Banerjee, "Image processing and data hiding framework on FPGA based platform", International Journal of Electrical, Electronics and Data Communication", Volume 1, PP. 77-82, May2013.

[57] S.S. Bedi, Rati Khandelwal, "Various Image Enhancement Techniques- A Critical Review", International Journal of Advanced Research in Computer and Communication Engineering (IJARCCE), ISSN (Online) 2278-1021, ISSN (Print) 2319-5940, Volume 2, Issue 3, PP. 1605-1609, March 2013.

[58] Priyanka Saini, Adesh Kumar, Neha Singh, "FPGA Implementation of 2D and 3D Image Enhancement Chip in HDL Environment", International Journal of Computer Applications, Volume 62, PP. 24-31, January 2013.

[59] Journal of Advanced Research in Computer Science and Software Engineering (IJARCSSE), ISSN 2277 128X, Volume 2, Issue 4, PP. 56-61, April 2012.

[60] Mrs. S. Allin Christe, Mr. M. Vignesh,Dr. A. Kandaswamy, "An efficient FPGA implementation of MRI , Image filtering \& Tumor characterization using Xilinx system generator", International Journal of VLSI design \& Communication Systems (VLSICS), Volume 2, PP. 95-109, December 2011

[61] Ruben Pasada-Gomez, Oscar Osvaldo Sandoval-Gonzalez, Albino Martinez Sibaja, Otniel Portillo-Rodri' griez, Giner Alor-Hernandez, "Digital image processing using LabVIEW", Practical Applications and Solutions Using LabVIEW Software, InTech, PP. 297-316, 1 august 2011.

[62] K. H. Fritzsche, M. Nolden, H-P. Meinzer, B. Stieltjes, "Diffusion Imaging in the Medical Imaging Interaction Toolkit (MITK)", Proc. Intl. Soc. Mag. Reson. Med. 19, PP. 4028, 2011.

[63] Simon Rit, Romulo Pinho, Vivien Delmon, Maxime Pech, Gauthier Bouilhol, Jo"el Schaerer, Bharath Navalpakkam, Jef Vandemeulebroucke, Pierre Seroul, and David Sarrut, "VV, a 4D slicer", Fourth International Workshop on Pulmonary Image Analysis, PP. 171-175, 2011. 


\section{Authors' Profiles}

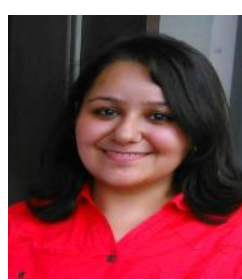

Garima Sharma has done M.Tech (ECE) from Department of Electronics and Communication Engineering (ECE), BPSMV Khanpur kalan, Sonipat, Haryana, India. She completed her B.Tech degree in Electronics and Communication from BPSMV Khanpur kalan, Sonipat, Haryana, India. Her area of interest includes Digital

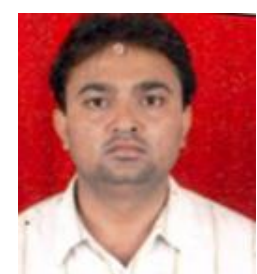

Krishan kumar is an assistant professor in Department of Electronics and Communication Engineering (ECE), BPSMV Khanpur kalan, Sonipat, Haryana, India. He received his B. E. in Electronics and Communication Engineering (ECE) from Chhotu Ram State College of Engineering, Murthal, Sonipat and M.Tech from Guru Jambheshwar University, Hisar, Haryana, India. His area of interest includes Image Processing and VLSI.

Image Processing.

How to cite this paper: Garima Sharma, Krishan Kumar,"Acceleration of Images via Software and Hardware using Proprietary Tools \& Open Sources for Healthcare Industry", International Journal of Image, Graphics and Signal Processing(IJIGSP), Vol.9, No.7, pp.10-22, 2017.DOI: 10.5815/ijigsp.2017.07.02 